\title{
Castleman's Disease of the Pleura: Case Presentation and Review of Literature
}

\author{
Pablo Loarte ${ }^{1}$, Nayle Araguez ${ }^{1}$, Rose Marie Flores ${ }^{1,2}$, Li-The Wu ${ }^{3}$, Ronny Cohen ${ }^{1,2^{*}}$ \\ ${ }^{1}$ Woodhull Medical Center, Brooklyn, USA; ${ }^{2}$ NYU School of Medicine, New York, USA; ${ }^{3}$ North Shore University Hospital, Forest \\ Hills, USA. \\ Email: *ronny.cohen@woodhullhc.nychhc.org
}

Received March 11 ${ }^{\text {th }}, 2012$; revised April 25 $5^{\text {th }}, 2012$; accepted May $16^{\text {th }}, 2012$

\begin{abstract}
Purpose: Castleman's Disease is a very rare medical disease and the presence of this condition in the pleura is even rarer and there are only few reported cases. The presence of symptoms is also unusual and has been mostly described in multicentric cases of castleman's disease instead of solitary lesions (unicentric castleman). We report a case of unicentric pleural castlema's disease with presence of constitutional symptoms. Methods: A 36-year-old male without any significant past medical history who presented to the emergency due to an incidental large pleural effusion seen in chest radiograph, but referring chronic constitutional symptoms consistent of significant weight loss and chronic cough. Results: Chest computed tomography revealed a mass and several attemps with minimally invase approach failed to identify the lesion. The mass was excised through VATS but due to its significant vascularity, it had to be converted to open thoracotomy. Conclusion: The treatment of unicentric lesions is mainly complete surgical excision and the prognosis is excellent with some chances of recurrence. There are less than one dozen of reported pleural castleman reported in the literature. This case evidence than unicentric lesion may exhibit constitutional symptoms and confirmed than lesions above $5 \mathrm{~cm}$ can be removed by VATS but the chances for conversion to an open thoracotomy are high.
\end{abstract}

Keywords: UCD: Unicentric Castleman's Disease; MCD: Multicentric Castleman's Disease; Pleural Effusion; VATS: Video Assisted Thoracoscopy; HIV: Human Immunodeficiency Virus

\section{Introduction}

Dr. Benjamin Castleman initially described this condition in 1956. Other names given to this condition are Angiofollicular lymph node hyperplasia or Giant lymph node Hyperplasia, Benign Lymphoma and Follicular Reticuloma [1,2]. Morphologically there are 2 forms of presentation; unicentric and multicentric [3]. The presence of Castleman disease in the pleura is extremely rare, because most of the thoracic Castleman lesions affect the mediastinum or hilia. This case presented with pleural effusion and not all the pleural castleman's lesions exhibit or present with pleural effusion [4]. The aim of this report is to report a very unusual case with a very uncommon presentation. Most reported cases of castleman's disease of the pleura did not exhibit constitutional symptoms and pleural effusion as we have in this case.

\section{Case Report}

A 36-year-old male patient presented to the emergency room after being referred by his primary care physician

${ }^{*}$ Corresponding author. due to an abnormal chest radiograph. He was complaining of chronic productive cough during the previous 2 months before admission. He also admitted night sweats and unintentional weight loss of approximately 30 pounds during a period of 8 months. He recently migrated from the Caribbean 6 months ago. He denied any significant past medical history or recent medication use.

On physical exam, he had a general good appearance. The head and neck examination did not reveal any lymphadenopathy and the cardiac exam was unremarkable. The lung examination showed absence of breath sound in the entire right lung field with absence of tactile fremitus but no intercostal retraction during inspiration. Abdominal exam was benign and the four extremities exhibited equal pulses without evidence of leg edema. Initial blood pressure (BP) 137/90 mmhg, heart rate (HR) $82 \mathrm{bpm}$, respiratory rate of $14 \mathrm{rpm}$, and Oxygen saturation of $98 \%$ in room air and temperature of $98 \mathrm{~F}$.

Initial laboratory evaluation was unremarkable (see Table 1). Mantoux test was placed and Sputum culture results were negative for Acid Fast Bacilli and mycobacterium species. 
Chest radiograph (Figure 1) showed a large right pleural effusion. Chest computed tomography (Figure 2) described a $7.5 \times 6.5$ by $7.2 \mathrm{~cm}$ mass in the inferior lateral margin of the right hemithorax, surrounded by large right pleural effusion, but the study was limited determining the anatomical origin of the mass.

Table 1. Initial laboratory results.

\begin{tabular}{cc}
\hline WBC & $6.29 / \mathrm{Ul}$ \\
\hline Hemoglobin & $17.0 \mathrm{~g} / \mathrm{dl}$ \\
Hematocrit & $49.9 \%$ \\
$\mathrm{MCV}$ & $88.3 \mathrm{fL}$ \\
Platelet & $235 / \mathrm{Ul}$ \\
$\mathrm{Na}$ & $141 \mathrm{mmol} / \mathrm{L}$ \\
$\mathrm{K}$ & $4.2 \mathrm{mmol} / \mathrm{L}$ \\
$\mathrm{BUN}$ & $12 \mathrm{mg} / \mathrm{dl}$ \\
$\mathrm{Creatinine}$ & $0.8 \mathrm{mg} / \mathrm{dl}$ \\
$\mathrm{Glucose}$ & 68 \\
Protein & $7.5 \mathrm{~g} / \mathrm{dl}$ \\
$\mathrm{Albumin}$ & $4.4 \mathrm{~g} / \mathrm{dl}$ \\
$\mathrm{ALT}$ & $17 \mathrm{U} / \mathrm{L}$ \\
$\mathrm{AST}$ & $20 \mathrm{U} / \mathrm{L}$ \\
$\mathrm{LDH}^{\mathrm{Ph}}$ & $330 \mathrm{U} / \mathrm{L}$ \\
$\mathrm{PaCO}$ & 7.43 \\
$\mathrm{PaO}$ & $37 \mathrm{~mm} \mathrm{Hg}$ \\
$\mathrm{HCO}_{3}$ & $74 \mathrm{mmHg}$ \\
Oxygen saturation & $24 \mathrm{mmol} / \mathrm{L}$ \\
\hline & $96 \%$ \\
\hline
\end{tabular}

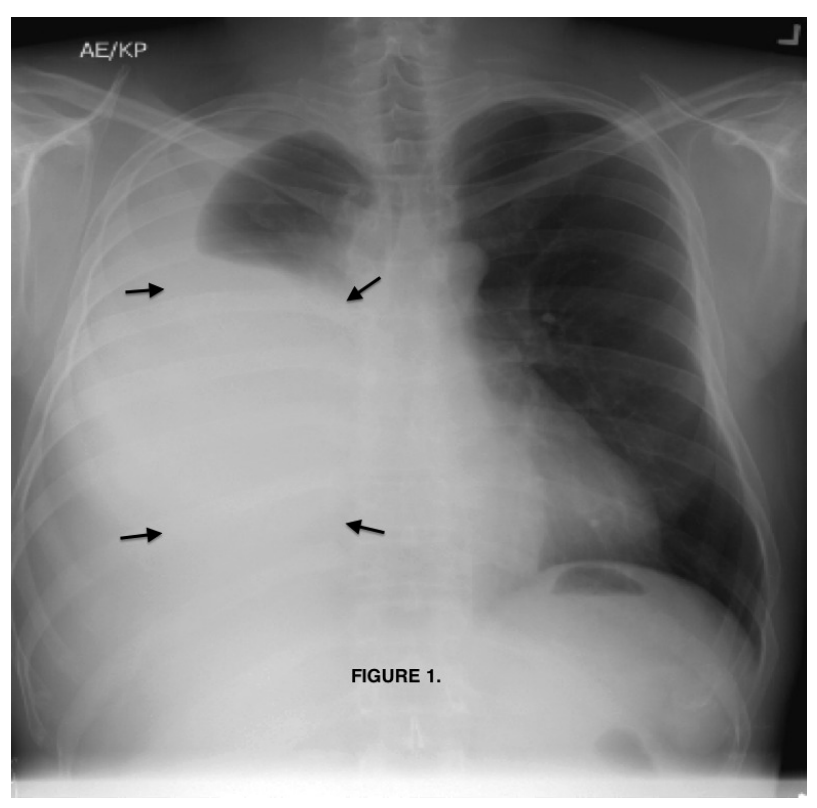

Figure 1. Chest radiograph, postero-anterior view with significant pleural effusion (arrows).

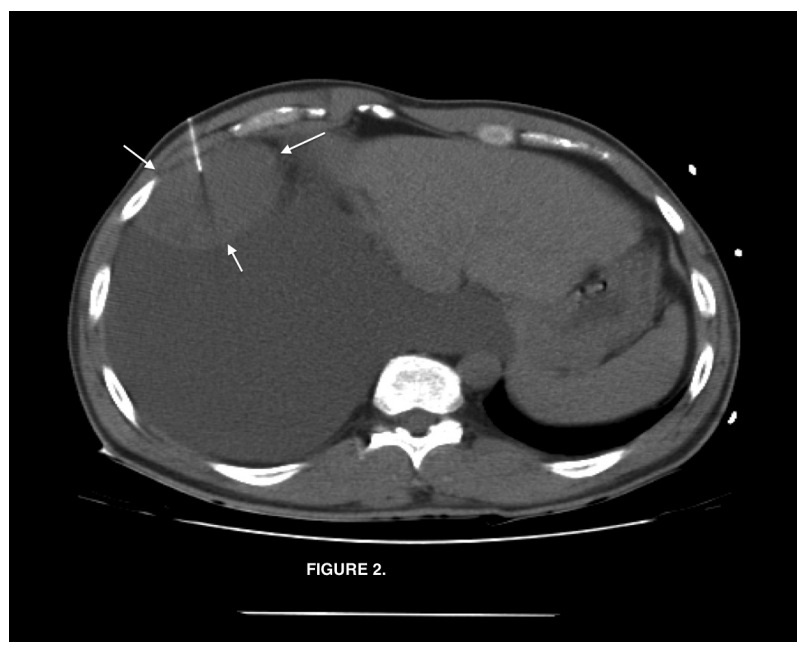

Figure 2. Chest computed tomography, axial view with evident mass in the inferior lateral margin of the right hemithorax, surrounded by large right pleural effusion (Arrows).

He was admitted for management of pleural effusion and further evaluation of lung mass. Right-sided thoracentesis was performed and 3.5 liters of slightly yellow turbid fluid was drained. The fluid was sent for analysis (see Table 2). A cytological report showed mixed B and T-cell population of lymphocytes. Flow cytometry and immunochemical analysis was performed (see Table 3).

Four days after first thoracentesis, the fluid re-accumulates and by day $6^{\text {th }}$ he developed dyspnea. Thoracentesis was repeated, obtaining 1.5 liters of fluid. He underwent Chest CT guided-FNA (fine needle aspiration) of the mass, but the result was non-diagnostic.

The decision was to perform a video assisted thoracoscopy (VATS) for resection of the mass. A sessile pleural parietal mass was identified in the phrenic angle, the phrenic nerve was free and two arteries were providing blood supply to the mass. The arterial supply was ligated and 2.5 liters of yellow fluid was drained from the pleural cavity during the VATS. The procedure was converted to a right thoracotomy due to persistent bleeding and the mass was excised by blunt dissection. He had two right-sided chest tube placed and admitted to Intensive care unit for observation.

The lesion was sent to the Hematopathology Department (Figures 3-7). Macroscopically was a sessile mass. Histologically evaluation showed a lymphoid mass composed of follicular proliferation with interfolicullar areas expanded by a proliferation of small vessels with hyalinization. The mantle zones were expanded with small to intermediate sized lymphocytes arranged predominantly in a concentric pattern "Onion Skinning", surrounding the atrophic germinal centers. Scattered plasma cells were seen mostly in the interfollicular regions. Immunohistochemical stains were performed and CD 20 high lighted 
Table 2. Initial pleural fluid analysis.

\begin{tabular}{cc}
\hline Fluid Analysis & Results \\
\hline RBC & $2600 / \mathrm{cu} \mathrm{mm}$ \\
WBC & $1090 / \mathrm{cu} \mathrm{mm}$ \\
Neutrophils & $1 \%$ \\
Lymphocytes & $96 \%$ \\
Monocytes & $3 \%$ \\
Glucose & $97 \mathrm{mg} / \mathrm{dl}$ \\
Cultures & No growth reported \\
Cytology & 7.56 \\
Ph & $121 \mathrm{U} / \mathrm{L}$ \\
LDH & $4.7 \mathrm{~g} / \mathrm{dL}$ \\
Protein
\end{tabular}

Table 3. Immunohistochemical analysis.

\begin{tabular}{cccccc}
\hline Marker & CD 3 & CD 20 & CD 10 & Bcl 2 & Bcl 6 \\
\hline Present + & + & $\begin{array}{c}\text { + In very small } \\
\text { subset of cells }\end{array}$ & - & $\begin{array}{c}\text { In a subset } \\
\text { of cells }\end{array}$ \\
Absent & & & Absent & \\
\hline
\end{tabular}

CD: Cluster of differentiation, $(+)$ : positive or present, $(-)$ : negative or absent, BCL 2: B-cell lymphoma 2 protein, BCL 6: B-cell lymphoma 6 protein.

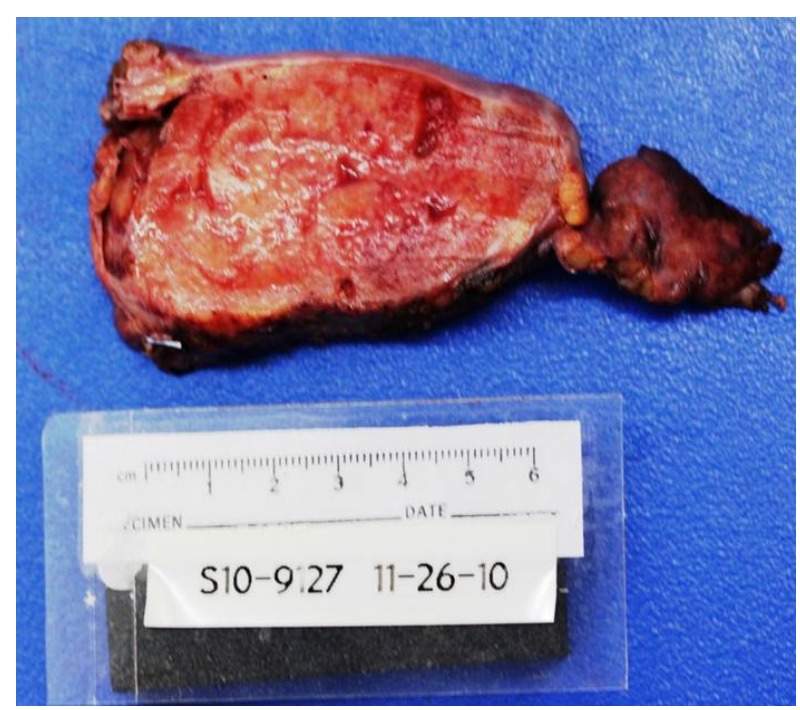

Figure 3. Macroscopic Castleman’s lesion.

in the atrophic germinal centers, CD 3 highlights T cells in the interfollicular area, CD 21 in the scattered follicular dendritic meshwork and CD 138 Highlighted the interfollicular plasma cells. The plasma cells were polyclonal for Kappa and light chain. Epstein Barr Virus was negative. These pathological findings were consistent with the diagnosis of Castleman Lymphadenopathy Hyaline Vascular type.

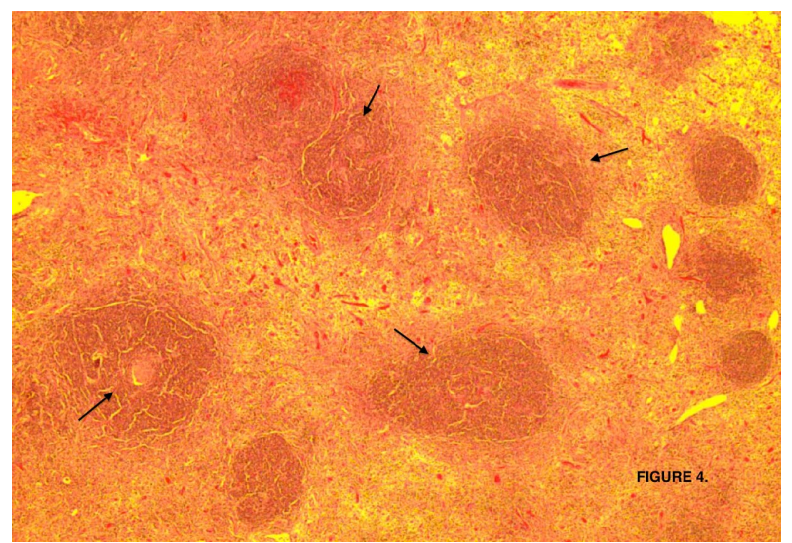

Figure 4. Lymphoid follicles with involuted and partly collagenized germinal centers (arrows).

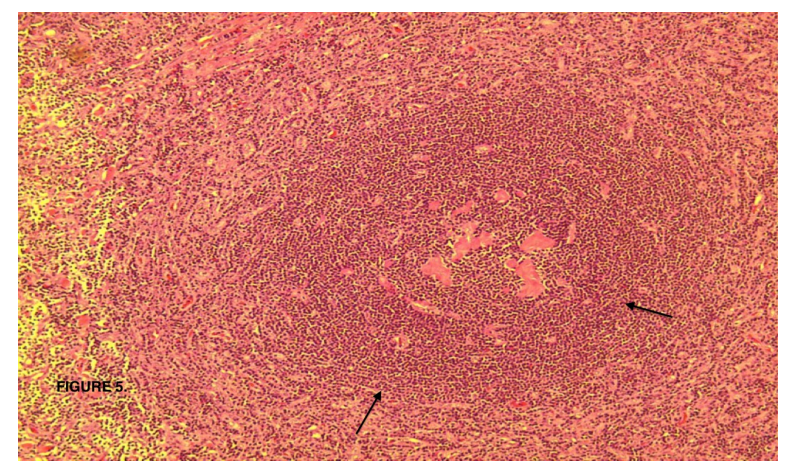

Figure 5. Lymphoid follicle (arrows) with involuted partly hyalinized germinal center.

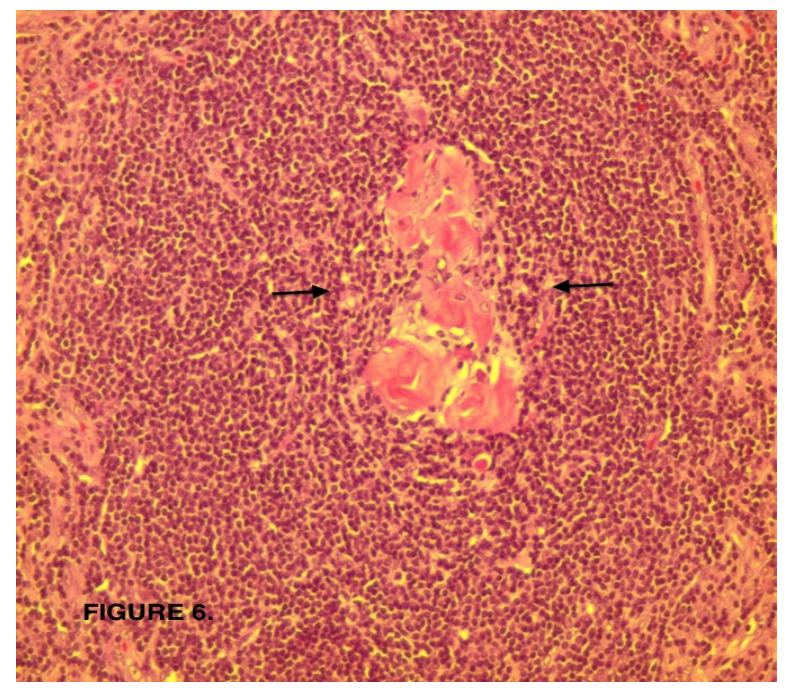

Figure 6. Hyalinized involuted (atrophic) germinal center (arrows).

Patient recovered uneventfully, his chest radiographs 1 and 2 months after the procedure did not show any evidence of pleural effusion recurrence and follow up Chest computed tomography at 1 year did not show any recurrence of previous lesion or a new one. 


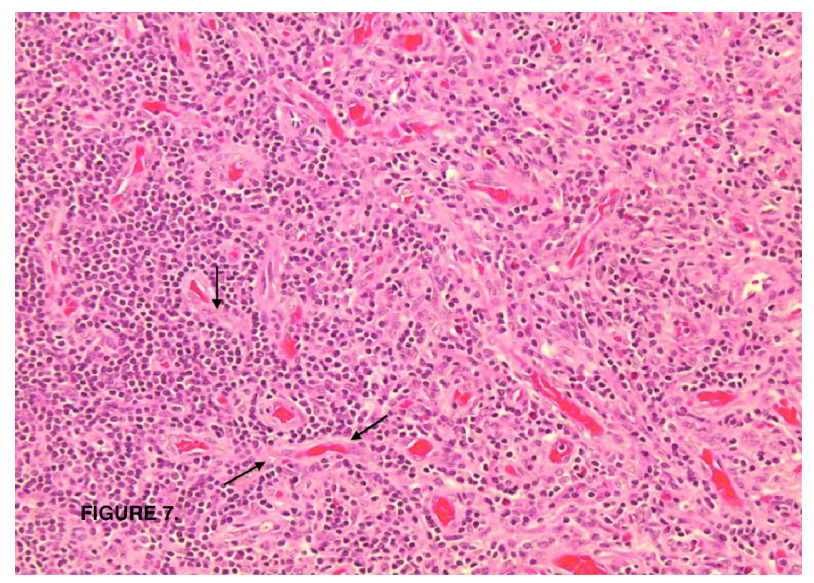

Figure 7. Interfollicular areas expanded by numerous blood vessels, some with hyalinization (arrows).

\section{Etiology}

Castleman's disease is a lymphoproliferative disorder characterized by enlarged hyperplastic lymph node(s) where there is a polyclonal B lymphocyte proliferation, often associated with autoimmune manifestations and connective tissue disease $[3,5,6]$. The etiology and pathogenesis is probably diverse. The role of inflammatory mediators like interleukin 6 (IL-6) has been described in Multicentric Castleman's disease (MCD) extensively but was also described in a unicentric case. Numerous cells produce IL-6, including B and T cells. IL-6 induces differentiation and proliferation. It is also involved in the generation of acute phase reactant proteins by the liver and this explains the presence of constitutional symptoms. A deregulated IL-6 gene expression has been observed only in affected lymph nodes of Castleman's Disease [5,7].

The vascular endothelial growth factor (VEGF) has been found to be elevated in lymph nodes of patients with Castleman; this can induce blood vessel proliferation $[5,8]$.

The B-lymphocytes express CD 20 in their surfaces; the suppression of CD 20 using Rituximab has shown to induce remission in $\mathrm{MCD}$ and reduction of size in a Unicentric lesion [5,9].

There is an extensively described relationship between viral diseases and Castleman's disease. Human Herpes Virus 8 (HHV-8) is a lymphotropic virus, etiologically linked to Kaposi sarcoma. The majority of the cases associated with HHV-8 infection happen in patients coinfected with HIV and the clinical presentation is as MCD. All the cases of HIV-associated Castleman are HHV-8 positive. A viral homolog, of IL-6 has been found to be involved in the activation of transcription pathways $[5,6,10]$.

Other viral agent associated is the Epstein-Barr virus (EBV) infection or HHV-4. In a retrospective study of 20 cases of Castleman, EBV was confirmed by PCR and ISH (in situ hybridization). EBV is also a lymphotropic virus associated with mononucleosis and some type of lymphomas. The mechanism by which EBV could possible cause Castleman is again linked to the increased production of IL- 6 by the virus [11-13].

Other conditions that have been found associated with $\mathrm{CD}$ are autoimmune disorders. Diseases like paraneoplastic pemphigus, rheumatoid arthritis, Evan's syndrome, amyloidosis, glomerulopathy and others more. It is though that Castleman and autoimmune disorders share many pathophysiologic features [3].

\section{Classification}

Morphologically is classified in Unicentric and Multicentric, based on the extent of lymph node involvement. There is a Histopathogenetic classification that divides it in 4 types: Hyaline Vascular, Plasma Cell, HHV 8-associated Castleman's disease and Multicentric Castleman disease not otherwise specified. Approximately 90\% of cases are Hyaline Vascular, occurring more in young adults. It is more common in unicentric than multicentric and is characterized by prominent vascularity in the germinal centers often with a single vessel, vascular proliferation between the follicles and the "onion-skinning" appearance of mantle zone $[1,13]$.

The plasma-cell type is commonly found in MCD, associated with systemic symptoms and abnormal laboratory findings. It represents less than $10 \%$ of the cases and has tendency to occur in older age groups $[1,13]$.

Th HHV-8 associated Plasmablastic Multicentric type has a poor prognosis with a survival of only months. It occurs mostly in immunosuppressed and HIV patients and has a risk of progression to a form of Large B-cell lymphoma [1,13].

The Multicentric Castleman disease not otherwise specified has a variable age of presentation. Most of the cases present with generalized lymphadenopathy, constitutional symptoms and hematologic/immunologic abnormalities. The diagnosis is of exclusion $[1,13]$.

\section{Diagnosis}

$70 \%$ of the cases occur in the chest, $15 \%$ in the neck, $15 \%$ in abdomen and pelvis, involving all of them primarily lymphatic tissues. Extralymphatic sites involved are the lungs, larynx, parotid glands, pancreas, meninges and muscles. Most of the reported cases are Unicentric in a $90 \%[1,10]$.

The disease is often asymptomatic, whether unicentric or multicentric. The MCD cases are more related with symptoms. The most commonly found are: fever, night sweats, weakness, fatigue, anorexia and significant weight loss. Disseminated lymphadenopathy is found in MCD 
and often associated with hepatosplenomegaly. Laboratory abnormalities commonly found include anemia, elevated erythrocyte sedimentation rate (ESR), proteinuria and polyclonal gammaglobulinemia [2,14-16].

On chest radiograph, mediastinal lesions do not differ from lesions like thymoma, lymphoma or solid tumors. Hiliar lesions may simulate bronchial adenomas and lung lesions present as lung mass. Massive pleural effusion can be an initial finding. Abdominal and pelvic lesions may not be visible on radiograph unless are calcified [17].

In computed tomography (CT), thoracic lesions can have 3 morphological patterns: solitary non invasive mass $(50 \%)$, dominant mass with involvement of contiguous structures (40\%) and matted lymphadenopathy confined to single mediastinal compartment. Contrast enhancement of the lesion reflects hypervascularity and this is considered a CT characteristic finding in either unicentric thoracic or abdominal lesion. Lesions larger than $5 \mathrm{~cm}$ can exhibit a heterogenous appearance [17].

In Positron emission tomography (PET) Castleman's lesions shows avid fluorodeoxyglucose (FDG) uptake. In cases of concurrent malignancy this can mimic metastatic lesion [1,18]. Magnetic resonance (MR) is good for evaluation; it can show tumor extension, relationship with adjacent structures and feeding vessels. Most of the lesions are isointense or slightly hyperintense relative to the skeletal muscle on T1-weighted images. Hyaline vascular type lesions classically exhibit heterogenous $\mathrm{T} 1$ and T2 hyperintensity. In cases which iodinated contrast material cannot be used, MR scanning is useful $[1,17$, 19].

The final diagnosis and identification is based on the pathological patterns.

\section{Treatment and Prognosis}

Cases of unicentric castleman's disease have a high cure rate with complete surgical excision. The chances of recurrence after surgical excision are rare; there are reported cases where the disease recurred after 1, 9, 18 and 20 years [5,20-23].

The surgical approach of unicentric lesion is variable, from thoracotomy to video assisted thoracoscopy (VATS) in thoracic lesions. Laparotomy or other approaches are indicated in intra-abdominal lesions. VATS can be safely performed and is minimally invasive; but patient's age, symptoms, tumor location and radiographic characteristics are important factors to evaluate before surgery. If necessary a conversion to open thoracotomy should be done [24-26].

Due to increased vascularization, tumor size or proximity to vital anatomical structures, alternative management with radiation, chemotherapy or vascular emboliza- tion can be used followed later by surgical excision. In a study published by de Vries et al. showed that $88 \%$ of those treated with radiation responded and in $43.8 \%$ of them the response was complete. Toxicities from radiotherapy can be acute or late and have been described. The embolization of feeding arteries is another option and agents like polyvinyl alcohol, gelfoam and microspheres can be used, but also complications exist. The use of chemotherapeutic agents to decrease tumor size is also evident in the literature. Rituximab has induced reduction in size and vascularity. Rituximab has been used in cases of multicentric HIV + Castleman disease [9,25,27].

The prognosis is good if unicentric. In the study published by Talat and Schulte, they divided all the published cases of Castleman's disease in 4 classes according to its pathological and morphological classification. The class III and I were the more predominant and correspond to the unicentric hyaline type and multicentric plasma cell type respectively. The 3-year disease free survival (DFS) rate was of $92.5 \%$ in the unicentric hyaline vascular type, $45.7 \%$ for HIV negative multicentric plasma cell type and $27.8 \%$ for HIV positive multicentric plasma cell type. The 3-year DFS was defined as: death resulting from disease or recurrence of disease [28].

\section{Discusion}

As mentioned above, Castleman's disease is very unusual and the presence of systemic symptoms in a unicentric hyaline vascular Castleman's disease is rare $[1,3,4]$. We had a young male who initially presented with constitutional symptoms. Many conditions were suspected, but the presence of Castleman was not in our differential diagnoses. Many case presentations were reviewed and very few of them had a remarkable symptomatology. Few cases of Hyaline vascular type who present with symptoms have been reported; the more commonly found are fever, night sweat and weight loss [29]. Our case is described in a young male and specifically talking about Pleural Castleman, in an exhaustive review of reported cases, most of them happened in females (See Table 4). This looks like there is a tendency to happen more in the female sex and it is more related to the histological type than to the anatomical location. In the study published by Talat and Schulte, they mentioned that females predominantly suffer from unicentric castleman and hyaline vascular type [28].

The pleural fluid analysis (See Table 2) shows a possible exudate. The fluid does not fully meet criteria for exudate because the ratio pleural/serum LDH is $<0.6$ and pleural LDH is not greater than $2 / 3$ of the upper limit normal serum value according to the Light's criteria. Using other classificatory methods, pleural LDH is not more than $130 \mathrm{U} / \mathrm{L}$ but our value is close to that number 
Table 4. Case reports of Pleural Castleman's disease. Among the 12 cases, only 2 were male and the rests, females. The predominant histological type was the hyaline vascular. HV: Hyaline vascular type and PC: Plasma cell type [4,32,33,35,36].

\begin{tabular}{cccc}
\hline \multicolumn{4}{c}{ Case Reports of Pleural Castleman's Disease } \\
\hline & Male & Female & Type \\
\hline Ko et al. & 1 & 7 & $6 \mathrm{HV}, 1 \mathrm{PC}$ and 1 Mixed type \\
Burrah et al. & 1 & $1 \mathrm{HV}$ \\
Ichiguchi et al. & 1 & $1 \mathrm{HV}$ \\
Reynolds et al. & 1 & & Unknown \\
O'Driscoll et al. & & 1 & $1 \mathrm{HV}$ \\
\hline
\end{tabular}

or near the cutoff [30].

The presence of pleural fluid lymphocytosis, pointed our differential diagnosis towards tuberculosis, lymphoma and sarcoidosis. The fluid cytology revealed a population of B and T-cells with Bcl-6 + in a subset of cells. Bcl-6 can be present on either healthy or neoplastic germinal centers of the B-cells. Antibody against Bcl-2 antigen is done along with Bcl-6 to distinguish neoplastic follicles from those found in benign hyperplasia [31].

In the CT, the diameter of this mass was about $7 \mathrm{~cm}$. In the literature, large lesions can be managed with VATS, but lesions above $5 \mathrm{~cm}$ of diameter showed severe adhesion and VATS had to be converted to open thoracotomy [32]. The pleural location is very unusual; question is if this is a location where lymph nodes are normally found. There are no lymph nodes in the pleural space, but subpleural space contains clusters of lymphoid cells and occasionally lymph nodes. The theory is that maybe intrapleural Castleman arises from the subpleura and growth toward the pleural space rather than into the lung $[32,33]$.

The presence of symptoms in unicentric type lesions is extremely unusual. In the Ko et al. report of 8 cases, 7 of them did not exhibit symptoms and only one case had dyspnea but was related to the massive pleural effusion. Our patient had a very florid clinical picture and we can hypothesize that IL-6 played a role. Unfortunately levels of IL-6 were no measured, but all the symptoms resolved after resection and 1 year after the excision there is no evidence of recurrence. The pathogenesis of pleural effusion in Castleman seems to be caused by local compression from the tumor [34].

\section{Conclusions}

Castleman's disease is a rare entity and pleural castleman is even more unusual. This case is very atypical if compared to the ones previously reported. It also confirms that the presence of symptoms, although is more prevalent in Multicentric type can be present in unicentric le- sions.

Based on this case experience and the extensive review of literature, it seems that lesions below $5 \mathrm{~cm}$ can be safely managed with minimally invasive approach like VATS and those whose size is above $5 \mathrm{~cm}$, have high chances to be converted to an open thoracotomy. In conclusion, the prognosis of unicentric lesions is excellent if total surgical excision can be achieved and appropriate lifetimes follow up should be instituted because there are although few, chances of recurrence. Due to the rarity of the condition and the few reported cases, it is still necessary to establish a cost-effective tool for following these cases.

\section{REFERENCES}

[1] D. Bornekamp, K. M. Horton, R. H. Hruban and E. K. Fishman, "Castleman Disease: The Great Mimic," $R a-$ dioGraphics, Vol. 31, No. 6, 2011, pp. 1793-1807. doi:10.1148/rg.316115502

[2] J. Herrada, F. Cabanillas, L. Rice, et al., "The Clinical Behavior of Localized and Multicentric Castleman Disease," Annals of Internal Medicine, Vol. 128, No. 8, 1998, pp. 657-662.

[3] T. W. Muskardin, B. A. Peterson and J. A. Molitor, "Castleman Disease and Associated Autoimmune Disease," Current Opinion in Rheumatology, Vol. 24, No. 1, 2012, pp. 76-83. doi:10.1097/BOR.0b013e32834db525

[4] S. P. Reynold, A. R. Gibbs, R. Weeks, et al., "Massive Pleural Effusion: An Unusual Presentation of Castleman's Disease," European Respiratory Journal, Vol. 5, No. 9, 1992, pp. 1150-1153.

[5] H. E. El-Osta and R. Kurzrock, "Castleman's Disease: From Basic Mechanism to Molecular Therapeutics," The Oncologist, Vol. 16, 2011, pp. 497-511. doi:10.1634/theoncologist.2010-0212

[6] B. Roca, "Castleman's Disease. A Review," AIDS Reviews, Vol. 11, 2009, pp. 3-7.

[7] K. Yoshizaki, T. Matsuda, N. Nishimoto, et al., "Pathogenic Significance of Interleukin-6 (IL-6/BSF-2) in Castleman's Disease," Blood, Vol. 74, No. 4, 1989, pp. 1360 1367.

[8] I. S. Abdul-Rahman, A. M. Al-Amri and K. Q. Ghallab, "Castleman's Disease: A Study of a Rare Lymphoproliferative Disorder in a University Hospital," Clinical Medicine: Blood Disorders, Vol. 2, 2009, pp. 5-19.

[9] B. Bandera, C. Ainsworth, J. Shikle, E. Rupard and M. Roach, "Treatment of Unicentric Castleman Disease with Neoadjuvant Rituximab," Chest, Vol. 138, No. 5, 2009, pp. 1239-1241. doi:10.1378/chest.09-2084

[10] A. Dham and B. A. Peterson, "Castleman Disease," Current Opinion in Hematology, Vol. 14, No. 4, 2007, pp. 354-359. doi:10.1097/MOH.0b013e328186ffab

[11] C. S. Restrepo, M. M. Chen, S. Martinez-Jimenez, J. Carrillo and C. Restrepo, "Chest Neoplasm with Infectious Etiologies," World Journal of Radiology, Vol. 3, No. 
12, 2011, pp. 279-288. doi:10.4329/wjr.v3.i12.279

[12] C. H. Chen, H. C. Liu, T. T. Hung and T. P. Liu, "Possible Roles of Epstein-Barr Virus in Castleman Disease," Journal of Cardiothoracic Surgery, Vol. 4, No. 31, 2009, pp. 1-5.

[13] D. M. Cronin and R. A. Warnke, "Castleman Disease. An Update on Classification and the Spectrum of Associated Lesions," Advances in Anatomic Pathology, Vol. 16, No. 4, 2009, pp. 236-246. doi:10.1097/PAP.0b013e3181a9d4d3

[14] H. J. Lachmann, J. A. Gilbertson, J. D. Gillmore, P. N. Hawkins and M. B. Pepys, "Unicentric Castleman's Disease Complicates by Systemic AA Amyloidosis: A Curable Disease," International Journal of Medicine, Vol. 95, No. 4, 2002, pp. 211-218.

[15] J. B. Alpert, D. Nonaka, A. Chachoua, et al., "Increasing Dyspnea Due to an Anterior Mediastinal Mass," Chest, Vol. 139, No. 1, 2011, pp. 217-223. doi:10.1378/chest.10-0383

[16] B. F. Menezes, R. Morgan, M. Azad, "Multicentric Catleman's Disease: A Case Report," Journal of Medical Case Reports, Vol. 1, No. 78, 2007.

[17] S. F. Ko, M. J. Hseih, S. H. Ng, et al., "Imaging Spectrum of Castleman's Disease," American Journal of Roentgenology, Vol. 182, No. 3, 2004, pp. 769-775.

[18] K. Enomoto, I. Nakamichi, K. Hamada, et al., "Unicentric and Multicentric Castleman's Disease," British Journal of Radiology, Vol. 80, No. 949, 2007, pp. e24-e26. doi:10.1259/bjr/93847196

[19] S. D. Chang and R. F. Thoeni, "Castleman's Disease Presenting as an Adnexal Mass: Ultrasound, CT and MRI Features," British Journal of Radiology, Vol. 77, No. 914, 2004, pp. 161-163. doi:10.1259/bjr/30098414

[20] K. Ohara, K. Narita, H. Ishikawa, et al., "Local Recurrence of Castleman Disease," Kyobu Geka, Vol. 62, No. 2, 2009, pp. 136-139.

[21] T. Ushio, K. Yoshimura and A. Kojima, "A Case of Castleman's Disease That Recurred Nine Years after Initial Surgical Removal," Nihon Kyobu Shikkan Gakkai Zasshi, Vol. 32, No. 12, 1994, pp. 1175-1180.

[22] N. Yaris, M. Cakir, M. Kalyoncu, et al., "Castleman Disease: A Case with Atypical Presentation," Pediatric Hematology Oncology, Vol. 21, 2004, pp. 203-208. doi:10.1080/08880010490276908

[23] H. Kurotaki, M. Kaimori and K. Nagai, "Recurred Castleman's Disease Containing a Fibrohistiocytic Nodular Lesion with Vascular Occlusion," Pathology International, Vol. 43, No. 10, 1993, pp. 603-607. doi:10.1111/j.1440-1827.1993.tb03237.x

[24] S. L. Han, X. X. Chen, X. F. Zheng, et al., "The Clinicopathological Behavior and Surgical Treatment of Ab- dominal Castleman's Disease," Singapore Medical Journal, Vol. 51, No. 10, 2010, pp. 813-816.

[25] S. Shetty, R. A. Brenes, L. Panait and J. Sanchez, "Video Assisted Thoracoscopic Resection of a Posterior Mediastinal Castleman's Tumor," Journal of Cardiothoracic Surgery, Vol. 6, No. 113, 2011.

[26] P. A. Seirafi, E. Ferguson and F. H. Edwards, "Thoracoscopic Resection of Castleman Disease, Case Report and Review," Chest, Vol. 123, 2003, pp. 280-282. doi:10.1378/chest.123.1.280

[27] I. A. C. De Vries, M. M. S. van Acht, et al., "Neoadjuvant Radiotherapy of Primary Irresectable Unicentric Castleman's Disease: A Case Report and Review of the Literature," Radiation Oncology, Vol. 5, No. 7, 2010.

[28] N. Talat and K. M. Schulte, "Castleman's Disease: Systematic Analysis of 416 Patients from the Literature," The Oncologist, Vol. 16, 2011, pp. 1316-1324. doi:10.1634/theoncologist.2011-0075

[29] P. Farruggia, A. Trizzino, N. Scibetta, et al., "Castleman's Disease in Childhood: Report of Three Cases and Review of the Literature," Italian Journal of Pediatrics, Vol. 35, No. 50, 2011.

[30] N. S. Paramothayan and J. Barron, "New Criteria for the Differentiation between Transudates and Exudates," Journal of Clinical Pathology, Vol. 55, No. 1, 2002, pp. 6971. doi:10.1136/jep.55.1.69

[31] A. S. Leong, K. Cooper and F. J. Leong, "Manual of Diagnostic Cytology," 2nd Edition, Greenwich Medical Media, Ltd., Cambridge, 2003, p. 29.

[32] S. F. Ko, S. H. Ng, M. J. Hsieh, et al., "Castleman Disease of the Pleura: Experience with Eight Surgically Proven Cases," Annals of Thoracic Surgery, Vol. 76, No. 1, 2003, pp. 219-224. doi:10.1016/S0003-4975(03)00133-4

[33] J. O’Driscoll, "Giant Lymph Node Hyperplasia Presenting as a Pleural Mass," Irish Medical Journal, Vol. 78, No. 5, 1985, pp. 132-133.

[34] M. G. Alexandrakis, F. H. Passam, D. Kyriakou and D. Bouros, "Pleural Effusions in Hematologic Malignancies,” Chest, Vol. 25, No. 4, 2004, pp. 1546-1555. doi:10.1378/chest.125.4.1546

[35] R. Burrah, V. Deshmane, S. Althaf, et al., "Castleman's Disease of the Pleura," General Thoracic and Cardiovascular Surgery, Vol. 59, No. 8, 2011, pp. 572-574. doi:10.1007/s11748-010-0676-x

[36] O. Ichiguchi and M. Yoshioka, "Castleman's Disease in the Cardiophrenic Angle Resected Thoracoscopically; Report of a Case," Kyobu Geka, Vol. 62, No. 2, 2009, pp. 161-163. 